\title{
A PC PROGRAM FOR COMPUTING CONFIDENCE BANDS FOR AVERAGE AND INDIVIDUAL GROWTH CURVES
}

\author{
Emet D. Schneiderman, ${ }^{*}$ Charles J. Kowalski, $†$ Stephen M. Willis* \\ and INGRID Y. GuO $\ddagger$ \\ *Department of Oral and Maxillofacial Surgery, Baylor College of Dentistry, Dallas, \\ TX 75266-0677, U.S.A.; †Department of Biologic and Materials Sciences and the Center for \\ Statistical Consultation and Research, University of Michigan, Ann Arbor, MI 48109, U.S.A.; \\ and $\ddagger$ Department of Public Health Sciences, Baylor College of Dentistry, Dallas, \\ TX 75266-0677, U.S.A.
}

(Received 18 June 1993; in revised form 20 October 1993; received for publication 9 November 1993)

\begin{abstract}
Dawson et al. (Am. J. Med. Genet. 7, 529-536, 1980) developed a procedure for constructing confidence bands for both average and individual growth curves which may be of considerable value in the study of growth and development. This paper describes their method for constructing, and provides a menu-driven GAUSS386i program for computing these confidence bands. It is demonstrated how these bands are useful for both the diagnosis and prognostication of growth patterns with known levels of confidence. It is assumed that the study is planned so that individuals will be measured at the same times, but missing data are allowed.
\end{abstract}

Longitudinal data Growth curves Confidence bands Diagnosis

\section{INTRODUCTION}

The construction of growth standards has commanded much attention in the biomedical literature (see e.g. [1-6]). This paper describes and implements a procedure for constructing confidence bands both for average and individual growth curves which was first used in [7] in a study of the development of head circumference in achondroplastic children. Given longitudinal measurements on the $N$ individuals comprising the normative or standardizing sample, polynomials are first fitted to the individual growth profiles and then confidence bands are constructed for the average growth curve (AGC), and for the growth curve of a "new" individual. The former indicates the accuracy with which the AGC has been estimated; the latter can be used to assess the likelihood that an individual is from the same population as the one from which the standardizing sample was drawn, i.e. for diagnostic purposes. It is assumed that the times of measurement for the individuals comprising the normative sample were planned to be the same, but missing values are allowed. The notation established in [8-12] is used. In particular, $D$ denotes the degree of the polynomial fit to a sequence of repeated measurements and $P=D+1$ the corresponding number of polynomial regression coefficients.

\section{THE STATISTICAL MODEL}

Consider $N$ individuals, each being measured at times $t_{1}, t_{2}, \ldots, t_{T}$ resulting in the vector of observations

$$
\mathbf{x}_{i}=\left[\begin{array}{c}
x_{i 1} \\
x_{i 2} \\
\vdots \\
x_{i T}
\end{array}\right] \text { for } i=1,2, \ldots, N
$$


We consider the case where the measurements are designed to be made at a common set of time points. Some of the $x_{i j}$ may be missing, but we assume that such missing data points are "missing at random", i.e. that occurrences of missing data are not related to the values of neighboring measurements [13]. We assume that a polynomial of some degree (to be determined) adequately fits the growth profile of each of the $N$ individuals. Dawson et al. [7] then propose to replace the observations, $\mathbf{x}_{i}^{\prime}=\left[x_{i 1}, x_{i 2}, \ldots, x_{i T}\right]$ for the $i$ th individual, by the estimated polynomial regression coefficients $\hat{t}_{i}=\left(\mathbf{W}_{i} \mathbf{W}_{i}\right)^{-1} \mathbf{W}_{i}^{\prime} \mathbf{x}_{i}$, where $W_{i}$ is the within-individual (time) design matrix specific to the $i$ th individual $[8,11]$.

Dawson et al. [7] then assume $\hat{t}_{i} \sim M V N(\tau, \Lambda)$, i.e. that $\hat{t}_{i}$ has a multivariate normal distribution with mean vector $\tau$ (the polynomial regression coefficients for the AGC) and covariance matrix $\Lambda$. Under this assumption, following [14, pp. 124-136], Dawson et al. [7] then used the fact that simultaneous $(1-\alpha) \times 100 \%$ confidence intervals for linear combinations of the form $\mathbf{a}^{\prime} \tau$ are given by

$$
\mathbf{a}^{\prime} \hat{\tau} \pm T_{a}(P, N-P) \sqrt{\frac{1}{N} \mathbf{a}^{\prime} \mathbf{S}(\hat{\tau}) \mathbf{a},}
$$

where $T_{\alpha}^{2}(P, N-P)=\{(N-1) P /(N-P)\} F_{1-\alpha}(P, N-P)$ and $F_{1-\alpha}(P, N-P)$ is the $(1-\alpha) \times 100$ th percentile of the $F$-distribution with $P$ and $N-P$ degrees of freedom.

This provides confidence bands for the ACG if we take $\mathbf{a}^{\prime}$, in succession, equal to

$$
\begin{gathered}
{\left[1, t_{1}, t_{1}^{2}, \ldots, t_{1}^{D}\right]} \\
{\left[1, t_{2}, t_{2}^{2}, \ldots, t_{2}^{D}\right]} \\
\ldots \\
{\left[1, t_{T}, t_{T}^{2}, \ldots, t_{T}^{D}\right] .}
\end{gathered}
$$

The probability that these intervals simultaneously trap the value of $\mathbf{a}^{\prime} \tau$ (the AGC) is $1-a$.

If we wish to develop analogous bands for an individual growth curve, say for a "new" individual with growth curve $\tau_{v}$, the limits in (2) need to be adjusted [7]. To compute a confidence interval for an individual observation, we must combine the variability that arises from the variation in the sample about the estimated AGC $(\Lambda)$ with the variability arising from uncertainty in the AGC itself $(\Lambda / N)$. Thus a set of $(1-\alpha) \times 100 \%$ confidence intervals for linear combinations $\mathbf{a}^{\prime} \tau_{v}$ are of the form

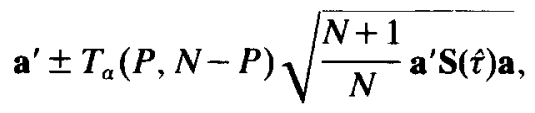

reflecting the fact that the variance of a predicted value of an individual observation is $\Lambda+\Lambda / N$. The reader may be more familiar with the distinction between the standard errors appropriate for predicting the mean and individual values (where the same switch between $1 / N$ and $(N+1) / N$ is made) in the context of simple linear regression analysis, $[15$, p. 30]. In any event, (4) gives confidence bands for an individual growth curve, again identifying $a^{\prime}$ with the values (3). The confidence bands for an individual may also be viewed as a set of what are known as prediction intervals in the classical regression literature $[16, p .70]$. This latter term reflects how these intervals might be used to predict whether a new subject is a member of the reference population. Prediction intervals resemble confidence intervals, but they differ conceptually. A confidence interval represents an inference on a parameter: a prediction interval is a statement about the value to be taken by a random variable.

It will be noted in the above that while different degree polynomials for individuals are possible in (2), the rest of the development assumes that $D(P)$ is the same for each 
individual. In [7], starting with a given $D, t$-tests of $H: \tau_{P}=0\left(\tau_{P}\right.$ is the coefficient of $\left.t^{D}\right)$ were provided for each individual. If this degree was required for one or more individuals, that $D$ was used to fit each of the $N$ growth profiles. Zerbe [17], when faced with the same problem, obtained $D_{1}, D_{2}, \ldots, D_{N}$ separately for each individual and used $D_{\max }$ for the common value of $D$, but instead of refitting, augmented for individuals having $D_{i}<D_{\max }$ with $D_{\max }-D_{i}$ zeros. Thus, e.g. if $D_{\max }=3$ and some $D_{i}=1$, a line was fit to the $i$ th profile and two zeros appended to $\hat{t}_{i}$ so that $\hat{t}_{i}$ is $4 \times 1$.

The results from the two approaches can differ considerably [18]; and neither is guaranteed to be satisfactory in every situation, so we allow both possibilities in our program. More details are given in $[19,20]$.

\section{IMPLEMENTATION}

In the previous section we outlined a method for constructing confidence bands for both the AGC and individual growth curves which allows individual-specific time design matrices. In this section we briefly describe the program implementing this method. More detailed descriptions are given in $[19,20]$. Information concerning obtaining a copy of the program, hardware requirements, etc., is given in the Appendix.

We ask that users of our program provide an $N \times T$ data matrix of the form (1), using periods (".") to signal missing data values. This data set may be in either ASCII or GAUSS format. Thus, for example, the data set considered in [7] woud be prepared as shown below:

$\begin{array}{ccccccccccccc}. & . & 39.4 & 41.9 & . & 42.5 & 43.8 & 45.7 & 46.9 & 47.6 & 48.3 & . & . \\ 34.3 & 36.8 & 41.2 & 42.5 & 43.8 & 45.7 & 46.3 & 47.6 & 48.9 & 49.5 & . & . & 50.0 \\ . & 41.0 & . & . & . & . & 47.0 & . & 50.0 & . & . & 53.0 & . \\ . & 40.0 & 42.0 & . & 44.5 & 45.5 & . & 48.0 & . & . & 49.8 & . & . \\ 38.7 & 40.0 & 41.2 & 43.1 & 45.1 & 45.7 & . & . & . & . & 50.2 & . & . \\ 36.8 & . & 40.3 & 41.5 & 42.3 & 43.8 & . & . & . & . & . & . & . \\ . . & 40.6 & 43.8 & 44.4 & 45.7 & 45.7 & 46.3 & 46.9 & 47.6 & 48.2 & 48.9 & 49.5 & 49.5 \\ 34.3 & 38.1 & 39.4 & 40.6 & 43.2 & 43.2 & 46.9 & . & . & . & . & . & . \\ . & 40.0 & 43.0 & . & 44.3 & 46.5 & 47.2 & . & 49.0 & . & 52.5 & . & 53.0 \\ . & 40.0 & 42.0 & 44.0 & 45.5 & . & 46.5 & . & . & . & . & . & . \\ . & . & . & . & . & . & . & . & 49.5 & 50.1 & 51.4 & . & 52.4\end{array}$

There are $N=11$ rows (individuals) and $T=13$ columns, the times of measurement being age in months from 0 to 12 . While there is a substantial amount of missing data, there is at least reasonable overlap of the growth profiles over the interval from 0 to 12 months. We have corrected an apparent typographical error in [7] where the observations at 10 and 11 months for the seventh individual are interchanged.

The program is invoked with the command gsruni zbands. The program menu then appears and the user is prompted for the location of the data file, which can be on a different drive (floppy or hard disk) or directory than the program itself. The indicated directory is searched and the names of those data files in either GAUSS or ASCII format are displayed. The user then highlights the file of choice using the cursor arrow keys and selects the file with the return key.

The user is then prompted for the type of data set: GAUSS or ASCII. We allow GAUSS data sets for the convenience of users who have installed GAUSS on their system. This is not necessary to run the program, which stands alone. Any program producing ASCII data sets can be used to create a file which will be accepted by our program. Next the values of $N$ and $T$ are provided. Finally, the confidence coefficient to be used in constructing the confidence bands is specified. Rather than simply computing the traditional $95 \%$ bands, we allow the user to make this choice consistent with the aims of the study. For example, if an obstetrician wanted to identify pregnant women whose fetuses were not growing according to expectation to the extent that additional prenatal 
care was indicated, he/she might want to set the confidence limits for the "normally growing population" lower (e.g. $90 \%$ ) so as to err on the side of falsely diagnosing "at risk" rather than as "within normal limits". This would be a particularly appropriate strategy if the benefit to risk ratio of the additional prenatal care was very high. One can also imagine situations in which one would want to set the confidence limits high. For example, if a manufacturer of eyeglasses for children wanted to provide broad coverage of frame sizes for all ages, perhaps they would choose $99 \%$ limits when doing studies on upper face and head dimensions.

The data are centered and the growth profiles for each of the $N$ individuals are plotted. We center the data, i.e. use the transformed time scale $t^{*}=t-\bar{t}$ where $\bar{t}$ is the mean of the time points, for computational accuracy as explained in $[19,20]$. The plots are made over the interval $[a, b]$, where $a=t_{1}$ and $b=t_{T}$. This is for the initial plot, whose purpose is to allow the user to make a reasonable guess at $D$ : The user has complete control over the final choice of $[a, b]$, as is illustrated in $[19,20]$. The user of this and related programs $[19,20]$ may choose between fitting all subjects with a single degree, $D$, or fitting each subject with a different degree polynomial, resulting in a sequence of degrees $D_{1}, D_{2}, \ldots, D_{N}$ which are individual-specific. After seeing the (unfitted) plots of the subjects' growth profiles, the user may select a single $D$ for all cases, and can then see what the fitted polynomial curves look like. If the user opts against a single $D$, he/she will then proceed to fit each case individually in an interactive fashion. With this latter choice, the vector of coefficients for each subject will be augmented with zeros so that $D_{i}=D_{\max }$ and these will then be used in the construction of the confidence bands. The details regarding these choices and the operation of this aspect of the program are discussed in $[19,20]$.

The output includes the values of the estimated polynomial regression coefficients for the individual growth curves, for the AGC, and plots of the corresponding confidence bands. Two sets of three plots each are provided: one set corresponds to the bands described in [12] and shown in [7]. These are occasionally called Scheffé-type bands. The second set is based on the use of the Bonferroni method for band construction which was mentioned in [7] but not considered in any detail. Seber [21, p. 83] and Timm [22], p. 166] describe this approach to confidence band construction. In bricf, the value $T_{\alpha}(P, N-P)$ in (2) and (4) is replaced by the value obtained from the ordinary, univariate $t$-distribuiton, $t_{a} *(N-1)$, the $100 \times(1-\alpha / 2 T)$ th percentile of the $t$-distribution with $N-1$ degrees of freedom. In the example considered in [7], we would use $\alpha^{*}=(1-0.05 / 26)=0.9981$, i.e. the 99.81 percentile of the $t$-distribution with 10 degrees of freedom. This would provide simultaneous $95 \%$ confidence bands for the curve at each of the $T=13$ times of measurement. The three plots for each type of confidence band (i.e. Scheffe and Bonferroni) are (i) the AGC with its confidence band, (ii) the confidence band for an individual (prediction interval), and (iii) the confidence band for the AGC and an individual shown together for contrast. The AGC is shown on all three plots to provide a frame of reference, but it should be noted that the individual confidence bands shown in (ii) and (iii) are for a hypothetical new member of the population, not the AGC.

\section{EXAMPLE}

Our example is based on the data considered above and in [7]. We illustrate the use of, and the output from, ZBANDS and compare our results with those in [7] which, as we have demonstrated $[19,20]$, are "flawed" due to the fact that $D=2$ was used for each individual, and this is inappropriate for case $\# 10$. While a quadratic fits the data for $\# 10$ very well over the interval he was observed, it extrapolates poorly to the interval of interest, 0 to 12 months. We have shown $[19,20]$ that when $D=1$ is used for this individual, the fit is reasonable over the entire period; and the result is tighter confidence bands than produced in [7]. Note that if $D=1$ (or some other degree) was not reasonable for \#10, the user has the option of deleting this case from the analysis $[19,20]$. 


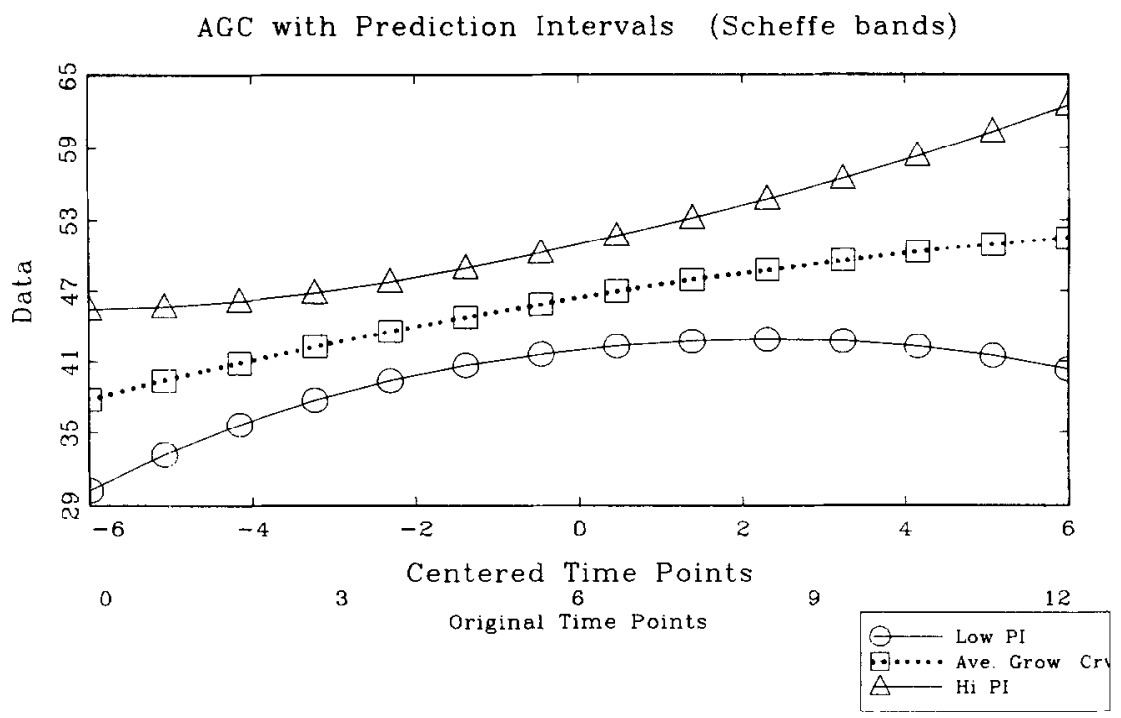

Fig. 1. Confidence bands for the individual, where $D=2$ for all subjects except \#10, where $D=1$. The original time points are at 1 -month intervals over the first year of life.

In any case, we obtain the following estimated polynomial regression coefficients (these are expressed in terms of the centered time points) as in [19]:

$\begin{array}{ccc}\hat{\tau}_{1} & \hat{\tau}_{2} & \hat{\tau}_{3} \\ 44.278 & 1.093 & -0.005 \\ 46.906 & 1.251 & -0.128 \\ 47.280 & 1.213 & -0.010 \\ 46.836 & 1.013 & -0.065 \\ 46.911 & 1.066 & -0.059 \\ 44.437 & 0.719 & -0.091 \\ 46.609 & 0.743 & -0.046 \\ 46.114 & 1.550 & -0.050 \\ 47.370 & 1.196 & -0.034 \\ 47.270 & 1.311 & 0 \\ 47.036 & 1.322 & -0.070\end{array}$

We next obtain the plots of the confidence bands. To conserve space we show only the plot for the Scheffé bands for individual growth curves in Fig. 1.

One might expect these bands to be somewhat narrower than those produced in [7], reflecting the reduced variability achieved by allowing case \#10 to be fit by a linear equation. This plot is shown in Fig. 2 (produced by our program, but using $D=2$ for case \#10).

The bands produced using $D=1$ for \#10 (Fig. 1) are somewhat narrower than when $D=2$ is used (Fig. 2); however, the difference is not dramatic, and is not uniform over the interval of observation. The band is narrower over approximately $69 \%$ of its length. Our program also produces tables like that shown below which can be used to compare the results in greater detail. Shown are the half-lengths of the confidence bands at each time of measurement. Again, as in Figs 1 and 2, we limit consideration to the Scheffé-type bands for the individual curves; but we add the results obtained when $\# 10$ is omitted from the analysis for comparison.

It is seen that the results for all three scenarios are broadly comparable, the most dramatic differences being between $D=1$ and $D=2$ for the later time points. It would appear that little is lost by omitting an individual from the analysis. These last two options may be preferable to "forcing" each subject to be fit by polynomials of the same degree. 


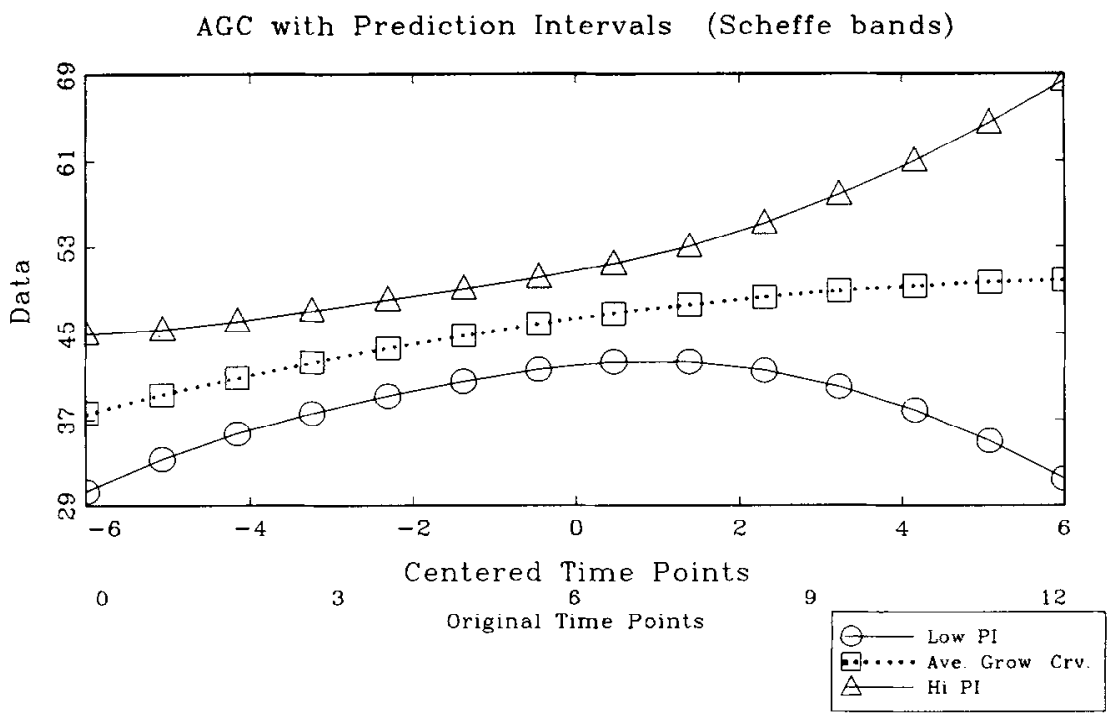

Fig. 2. Confidence bands for the individual where $D=2$ for all subjects. Note that the bands are at most times broader than those in Fig. 1.

\section{DISCUSSION}

We have outlined a method for constructing confidence bands for both the AGC and individual growth curves which allows individual-specific time design matrices. This method was implemented so as to accept data sets of the form (1) which contain missing observations.

The method and program were illustrated using data for which common times of measurement were planned, but a substantial amount of missing data ensued. It was noted, in this example, that somewhat tighter confidence bands resulted when individuals were allowed to have differing degrees of polynomials fit to their growth profiles, since this mitigated a problem with extraneous variability arising when one of the curves was extrapolated beyond the corresponding observation period. The confidence bands for the AGC generated by this method are analogous to those yielded by our programs that implement Rao's one-sample polynomial growth curve model $[8,12]$ and the twostage model [11] in that they reflect the precision with which the AGC was estimated. For example, a $99 \%$ confidence band has the explicit interpretation that one is $99 \%$ confident that the true (population) AGC is within these bands. The difference between the current approach and $[8,11,12]$ is that these earlier methods are more structured, requiring complete data, and/or making specific assumptions about the covariance

Table 1. Half-lengths of Scheffé-type confidence bands under different strategies for dealing with individual \#10.

\begin{tabular}{lccc}
\hline Time point & $\begin{array}{c}\text { Length when } \\
D=2 \text { for \#10 }\end{array}$ & $\begin{array}{c}\text { Length when } \\
D=1 \text { for } \# 10\end{array}$ & $\begin{array}{c}\text { Length when } \\
\# 10 \text { is omitted }\end{array}$ \\
\hline 0 & 7.334 & 7.606 & 8.101 \\
1 & 6.044 & 6.246 & 6.683 \\
2 & 5.230 & 5.215 & 5.606 \\
3 & 4.756 & 4.527 & 4.889 \\
4 & 4.469 & 4.178 & 4.524 \\
5 & 4.287 & 4.124 & 4.466 \\
6 & 4.250 & 4.306 & 4.647 \\
7 & 4.532 & 4.677 & 5.009 \\
8 & 5.351 & 5.220 & 5.527 \\
9 & 6.813 & 5.944 & 6.206 \\
10 & 8.904 & 6.873 & 7.067 \\
11 & 11.57 & 8.030 & 8.138 \\
12 & 14.77 & 9.436 & 9.445 \\
\hline
\end{tabular}


structure of the repeated measurements. The approach we outline here is much less stringent in these regards.

The confidence bands for an individual curve can be used to make an explicit statement, with a known level of confidence, as to whether the individual is a member of a reference population with regard to growth pattern. For example, a clinical investigator may wish to know whether mandibular growth is "normal" in a 7-year-old girl who has juvenile rheumatoid arthritis (JRA); this disease often affects the temporomandibular joint, leading to deficient lower facial growth. Using a longitudinal sample of healthy girls from 5 to 15 one can construct a $95 \%$ confidence band for individual "normal" children. If one or more measurements of the child with JRA were below this band, one might reasonably conclude that this child is not a part of the normal population. With this knowledge of disturbed mandibular growth, the clinician might consider initiating therapeutic intervention in which the afflicted joint is targeted, rather than waiting for the facial deformity to fully manifest itself several years later.

Two different kinds of bands are produced for each of the curves: Scheffé-type and Bonferroni. For a given $N$, widths of the former depend on the degree of the polynomial(s) fit (through $P=D+1$ in equation 2); the higher the degree, the wider the confidence bands. (For fixed $N$, in equation $(2), F_{1-a}(P, N-P)$ decreases with increasing $P$ but $(N-1) P /(N-P)$ increases at a faster rate.) The widths of the Bonferroni bands depend on $T$, the number of points at which the bands are constructed. The larger the value of $T$, the wider the bands. We should also note that while Dawson et al. [7] found the Scheffé-type confidence bands to be narrower for their data, the Bonferroni-based bands can be narrower, especially in situations where the degree of the polynomial fit is closer to the number of time points studied. For example, if the data in [7] were based on $T=5$ time points, the Bonferroni bands would be considerably narrower. We include both in our program for the convenience of potential users.

\section{SUMMAR Y}

Dawson et al. [7] developed a method for constructng confidence bands for both average and individual growth curves which may be of considerable value in the study of growth and development. We describe their method for constructing, and provide a GAUSS386i program for computing these confidence bands. In particular, given assumed multivariate normally distributed longitudinal observations on each of a sample of individuals from a given population, we show how to derive a prediction region with the property that one has a given confidence that the next observation from that population will fall into it. The times of measurement of the longitudinal observations for the standardizing sample need not be equally spaced and missing data are allowed. We assume that the study was planned to have common times of measurement, but allow some measurement sequences to be incomplete.

The method and the use of the program were illustrated using an example with a substantial amount of missing data. We discussed how these confidence intervals might be used by biomedical investigators and clinicians to diagnose and prognosticate, i.e. determine an individual's current growth status (e.g. is he/she growing normally?) as well as anticipate his/her future growth pattern with a known level of confidence.

Acknowledgement-Supported by grant DE-08730 from the National Institute of Dental Research.

\section{REFERENCES}

1. H. Goldstein, The construction of standards for measurement subject to growth, Hum. Biol. 44, 255-261 (1972).

2. H. Goldstein, The Design and Analysis of Longitudinal Studies. Academic Press, New York (1979).

3. M. J. R. Healy, Notes on the statistics of growth standards, Ann. Hum. Biol. 1, 41-46 (1974).

4. M. J. R. Healy, Statistics of growth standards, Human Growth, Vol. 1, Principles and Prenatal Growth, F. Falkner and J. M. Tanner, Eds. Plenum, New York (1978).

5. J. M. Tanner, Some notes on the reporting of growth data, Hum. Biol. 23, 93-159 (1951).

6. J. M. Tanner, The assessment of growth and development in children, Arch. Dis. Child. 27, 10-33 (1952).

7. D. V. Dawson, A. B. Todorov and R. C. Elston, Confidence bands for the growth of head circumference in achondroplastic children during the first year of life, Am. J. Med. Genet. 7, 529-536 (1980). 
8. E. D. Schneiderman and C. J. Kowalski, Implementation of Rao's one-sample polynomial growth curve model using SAS, Am. J. Phys. Anthrop. 67, 323-333 (1985).

9. E. D. Schneiderman and C. J. Kowalski, Implementation of Hills' growth curve analysis for unequal-time intervals using GAUSS, Am. J. Hum. Biol. 1, 31-42 (1989).

10. E. D. Schneiderman, C. J. Kowalski and T. R. Ten Have, A GAUSS program for computing an index of tracking from longitudinal observations, Am. J. Hum. Biol. 2, 475-490 (1990).

11. T. R. Ten Have, C. J. Kowalski and E. D. Schneiderman, PC program for analyzing one-sample longitudinal data sets which satisfy the two-stage polynomial growth curve model, Am. J. Hum. Biol. 3 , 269-279 (1991).

12. E. D. Schneiderman, S. M. Willis, T. R. Ten Have and C. J. Kowalski, Rao's polynomial growth curve model for unequal-time intervals: A menu-driven GAUSS program., Int. J. Biomed. Comput. 29, 235-244 (1991).

13. D. B. Rubin, Inference and missing data, Biometrika 63, 581-592 (1976).

14. D. F. Morrison, Multivariate Statistical Methods, 2nd Edn. McGraw-Hill, New York (1976)

15. N. Draper and H. Smith, Applied Regression Analysis, 2nd Edn. Wiley, New York (1981).

16. J. Neter and W. Wasserman, Applied Linear Statistical Models. Irwin, Homewood, IL (1974).

17. G. $O$. Zerbe, A new nonparametric technique for constructing percentiles and normal ranges for growth curves determined from longitudinal data, Growth 43, 263-272 (1979).

18. T. R. Ten Have, C. J. Kowalski and E. D. Schneiderman, A PC program for obtaining orthogonal polynomial regression coefficients for use in longitudinal data analysis, Am. J. Hum. Biol. 4, 403-416 (1992).

19. E. D. Schneiderman, S. M. Willis and C. J. Kowalski, PC program for estimating polynomial growth, velocity, and acceleraton curves when subjects may have missing data, Int. J. Biomed. Comput. 33, 249265 (1983).

20. E. D. Schneiderman, S. M. Willis and C. J. Kowalski, Clustering on the basis of longitudinal data, Comp. Biol. Med. 23, 399-406 (1993)

21. G. A. F. Seber, Multivariate Observations. Wiley, New York (1984).

22. N. H. Timm, Multivariate Analysis with Applications in Education and Psychology. Wadsworth, Belmont, CA (1975).

\begin{abstract}
About the Author-Emet D. Schnejderman received a B.A. and M.A. in Anthropology from Northwestern University in 1978, and the Ph.D. in Biological Anthropology from the University of Michigan in 1985. While at the University of Michigan he was affiliated with the Center for Human Growth and Development and began conducting research in the area of craniofacial growth. In collaboration with Joseph Mudar, Schneiderman developed an integrated software system for the analysis of cephalometric radiographs (X-rays of the head). While on the orthodontics faculty of the University of Detroit School of Dentistry from 1985 to 1988 , Dr Schneiderman created a computerized cephalometry laboratory. In 1988 Dr Schneiderman went to the Baylor College of Dentristy in Dallas where he is the director of research for the Department of Oral and Maxillofacial Surgery and Pharmacology Sciences. Dr Schneiderman and co-investigator Dr Charles Kowalski have been funded by NIH/NIDR from 1988 to 1993 to conduct this biostatistical research from which this paper issued. Dr Schneiderman has more than 50 publications including two chapters and the monograph, Facial Growth in the Rhesus Monkey, Princeton University Press (1992).
\end{abstract}

About the Author-Charles J. Kowalski received the B.S. in Mathematics from Roosevelt University in Chicago in 1962, the M.S. in Statistics from Michigan State University in 1965, and the Ph.D. in Biostatistics from The University of Michigan in 1968. Dr Kowalski then joined the faculty of the Department of Oral Biology at the Univesity of Michigan School of Dentistry. Dr Kowalski served as the assistant director of the university's Statistical Research Laboratory from 1971 to 1978 and research scientist at the Dental Research Institute from 1978 to the present and directed the institute's biometrics laboratory. He has been full professor of dentistry and statistician at The University of Michigan since 1978. At various times Dr Kowalski has served as a consultant to the National Football League, Park, Davis and Co., Nymegen University, Lancaster Cleft Palate Clinic, the Department of Antiquities of the University of Alexandria in Egypt, the U.S. Veterans Administration and the Eastman Dental Center. Dr Kowalski has published more than 200 scientific papers, including numerous chapters and the book $A$ Mixed-Longitudinal Interdisciplinary Study of Growth and Development published by Academic Press in 1979. Dr Kowalski's research has focused on the application of statistical methods to dental and oral resarch with special emphasis on measurement processes, their validity, reliability and calibration. Longitudinal data analysis and the computer implementation of polynomial growth curve models have also been and continue to be a major thrust of his research. Drs Kowalski and Schneiderman have been funded by NIH/NIDR from 1988 to 1993 to study and implement biostatistical methods for the analysis of longitudinal data in the form of user-friendly microcomputer programs.

About the Author-STEPHEN M. WILlis received the B.S. degree in Mathematics from the University of Texas at Arlington in 1987. Mr Willis has over 15 years of experience in cinical toxicology and is currently operations manager of a regional toxicology laboratory in Dallas. $\mathrm{Mr}$ Willis is also the lead programmer/systems analyst for the NIH/NIDR grant on longitudinal statistical methods with Drs Kowalski and Schneiderman. Mr Willis has played a major role in 
the development of user-friendly interfaces for programs that have broad applications in the biomedical sciences.

\begin{abstract}
About the Author-INGRID Y. Guo received a B.S. in Physics in 1982 from National Kaohsiung Teachers' College in the Republic of China. In 1988, she received an M.S. in Mathematics from The Ohio State University. She received her Ph.D in 1992 from The University of Southwestern Louisiana. Her dissertation topic was "Decision theoretic point estimation in a multivariate normal distribution". Dr Guo has been a statistical consultant for over 4 years. She joined the Baylor College of Dentistry as statistician and assistant professor of public health sciences in 1991. She has strong interests in applied as well as mathematical statistics. She is currently the statistician on an NIH grant funded from 1993 to 1996. Dr Guo is also the statistician for several grants funded by private industry. Her major research interests are decision theory/analysis, longitudinal analysis, meta-analysis, and experimental design. As a young researcher, she has published eight scientific papers.
\end{abstract}

\title{
APPENDIX: COMPUTER IMPLEMENTATION
}

The full set of PC programs for longitudinal data analysis can be obtained on $5.25^{\prime \prime}$ or $3.5^{\prime \prime}$ diskettes (please request type) by sending $\$ 25$ to defray the cost of handling and licensing fees. These programs require a 80386 or 80486 based personal computer (PC) running the MS-DOS operating system (version 5.0 or higher is recommended, although versions as low as 3.3 will suffice). 80386 computers must also be equipped with a 80387 math coprocessor. At least $4 \mathrm{mb}$ of memory is required, and must be available to GAUSS386i, i.e. not in use by memory resident programs such as Windows. Five mb of hard disk space are required to display the color graphics; VGA or SVGA is suggested to display optimally the graphic results. Runtime modules are supplied with the programs so that no additional software (i.e. compiler or interpreter) is required to run these programs. One can create and edit ASCII data sets for use by these programs using the full-screen editor supplied with MS-DOS version 5.0. The programs are written and compiled using GAUSS386i (version 3.01, release 14), require no additional installation or modification, and are run with a single command. When requesting the programs, address inquiries to the corresponding author and makes checks payable to Baylor College of Dentistry. 